\title{
Inhibition of p38 MAP kinase pathway induces apoptosis and prevents Epstein Barr virus reactivation in Raji cells exposed to lytic cycle inducing compounds Giulia Matusali1 ${ }^{1}$, Giuseppe Arena1 ${ }^{1}$ Alessandra De Leo ${ }^{1}$, Livia Di Renzo ${ }^{2}$ and Elena Mattia*1
}

Address: ${ }^{1}$ Dept. of Public Health Sciences, University "La Sapienza", P. le A. Moro 5, 00185 Rome, Italy and ${ }^{2}$ Dept. of Experimental Medicine and Pathology, University "La Sapienza", V. le Regina Elena 324, 00161 Rome, Italy

Email: Giulia Matusali - giuliamat@hotmail.com; Giuseppe Arena - giusare@yahoo.it; Alessandra De Leo - Alessandra.Deleo@alice.it; Livia Di Renzo - livia.direnzo@uniroma1.it; Elena Mattia* - Elena.Mattia@uniroma1.it

* Corresponding author

Published: 9 March 2009

Molecular Cancer 2009, 8:18 doi:10.1 I86/1476-4598-8-18

This article is available from: http://www.molecular-cancer.com/content/8/I//8

(c) 2009 Matusali et al; licensee BioMed Central Ltd.

This is an Open Access article distributed under the terms of the Creative Commons Attribution License (http://creativecommons.org/licenses/by/2.0), which permits unrestricted use, distribution, and reproduction in any medium, provided the original work is properly cited.
Received: 7 November 2008

Accepted: 9 March 2009

\begin{abstract}
Background: EBV lytic cycle activators, such as phorbol esters, anti-immunoglobulin, transforming growth factor $\beta$ (TGF $\beta$ ), sodium butyrate, induce apoptosis in EBV-negative but not in EBV-positive Burkitt's lymphoma (BL) cells. To investigate the molecular mechanisms allowing EBV-infected cells to be protected, we examined the expression of viral and cellular antiapoptotic proteins as well as the activation of signal transduction pathways in BL-derived Raji cells exposed to lytic cycle inducing agents.

Results: Our data show that, following EBV activation, the latent membrane protein I (LMPI) and the cellular anti-apoptotic proteins MCL-I and BCL-2 were quickly up-regulated and that Raji cells remained viable even when exposed simultaneously to $P(B U)_{2}$, sodium butyrate and TGF $\beta$. We report here that inhibition of p38 pathway, during EBV activation, led to a three fold increment of apoptosis and largely prevented lytic gene expression.

Conclusion: These findings indicate that, during the switch from the latent to the lytic phase of EBV infection, P38 MAPK phosphorylation plays a key role both for protecting the host cells from apoptosis as well as for inducing viral reactivation. Because Raji cells are defective for late antigens expression, we hypothesize that the increment of LMPI gene expression in the early phases of EBV lytic cycle might contribute to the survival of the EBV-positive cells.
\end{abstract}

\section{Background}

Epstein Barr Virus (EBV), the causative agent of infectious mononucleosis, is associated with an increasing number of malignancies of epithelial and lymphoid origin that include Burkitt's lymphoma (BL), nasopharyngeal carcinoma, Hodgkin's lymphoma and immunoblastic lymphomas in posttransplant and AIDS patients [1].
Following primary infection, EBV infects epithelial cells where it undergoes lytic replication, and B cells, where it usually maintains a latent state [2]. All EBV-associated tumors have a predominantly latent pattern of viral gene expression. Three types of latent programs have been characterized depending on the differential expression of a limited set of viral genes. These include six nuclear anti- 
gens (EBNA1, 2, 3A, 3B, 3C and LP) and three membraneassociated proteins (LMP1, LMP2A and 2B) plus several small RNA species (EBERs).

In vitro, EBV infection of peripheral B lymphocytes results in their immortalization and continuous proliferation [3]. Among the latent proteins, LMP1 plays a prominent role in the process of EBV-associated oncogenesis. This integral membrane protein can cause transformation of rodent fibroblasts and epithelial cells in vitro $[4,5]$ and induce development of B cell lymphoma or epidermal hyperplasia in transgenic mice $[6,7]$. By functioning as constitutively activated member of the tumor necrosis factor receptor (TNFR) family, through the cytoplasmic carboxy terminus LMP1 triggers several signaling pathways to alter cell growth and survival $[8,9]$. This viral oncoprotein stimulates NFkB, JNK, the JAK/STAT, PI3K/Akt, ERK1/2, and p38 mitogen activated protein kinase (MAPK) signal transduction cascades [10]; in addition, it regulates several downstream genes including anti-apoptotic genes such as bcl-2 [11,12], mcl-1 [13], A20 [14] and survivin [15].

Viral reactivation is initiated by the two immediate early proteins BZLF1 (ZEBRA or Zta) and BRLF1 (Rta) $[16,17]$ that function as transcriptional activators of EBV early genes [18-20].

In vitro, latency can be disrupted by a variety of different agents such as phorbol esters, sodium butyrate, TGF $\beta$, anti-immunoglobulins (anti-IgG) and calcium ionophores [21-23].

It has been reported that all these compounds induce apoptosis in EBV-negative cells but not in BZLF1-positive cells that appeared to be protected. Moreover, the antiapoptotic effect was prevented by treatment of the cells with inhibitors of viral DNA synthesis, leading to the hypothesis that a late EBV gene product might be responsible for survival of EBV-positive cells exposed to lytic cycle inducing compounds [24].

In this report we have further examined the connection between EBV lytic cycle induction and survival of the host cell aiming to detect viral gene products and/or signal transduction pathways involved in the protective effect. To focus on the early phases of EBV productive cycle, we used Burkitt lymphoma-derived Raji cells that, because of a deletion in EBV genome, support an abortive cycle, only allowing immediate early (IE) and early (E) genes expression [25]. We have previously shown that treatment of Raji cells with phorbol-12,13-dibutyrate $\left(\mathrm{P}(\mathrm{BU})_{2}\right)$, sodium butyrate and TGF $\beta$, activates EBV lytic cycle in more than $60 \%$ of the cell population [26]. We report here that following EBV activation, LMP1 and bcl-2 were promptly up-regulated and, despite the lack of viral late products, Raji cells were protected from apoptosis. We demonstrate that the suppression of $\mathrm{p} 38$ phosphorylation by its specific inhibitor caused a three fold increment of apoptosis. Furthermore, we found that inhibition of $\mathrm{p} 38$ signaling pathway largely prevented EBV lytic gene expression. These findings indicate that p38 MAPK plays a key role both in EBV activation as well as in host cell survival. In addition, the increment of LMP1 expression at the onset of the lytic phase suggests that the latent viral oncogene might contribute to preserve cell viability in the early stages of EBV productive cycle.

\section{Results}

Activators of EBV lytic cycle induce apoptosis in EBVnegative Burkitt's lymphoma cells

EBV lytic cycle can be induced in Burkitt's lymphoma cells by treatment with phorbol esthers, anti-immunoglobulin, sodium butyrrate or TGF $\beta$. Although each of these agents cause apoptosis, it has been reported that lytic EBV gene expression prevents the cells to undergo the programmed cell death [24].

To further investigate the protective effect exerted by EBV infection, we treated EBV-positive Raji and EBV-negative Ramos and BL41cells with $\mathrm{P}(\mathrm{BU})_{2}$, sodium butyrate and TGF $\beta$, a combination of agents able to trigger, in the former, EBV immediate early (IE) and early antigen (EA) expression in more than $60 \%$ of the population [26]. In the cells exposed to these compounds, we monitored apoptosis by analyzing in a cytofluorimeter the sub-G1 events and by Western blotting the proteolytic cleavage of poly(ADP-ribose) polymerase (PARP). Fig. 1A illustrates the results of a representative experiment where the percentages of cells with a sub-G1 DNA content were measured in the samples collected after 24 and 48 hours of incubation with EBV lytic cycle inducing agents. It appears that the fraction of EBV-negative cells with an hypodiploid DNA content increased with the incubation time. In particular, after 48 hour, about $50 \%$ of BL41 and $70 \%$ of Ramos cells were found in the sub-G1 peak. In contrast, treatment of EBV-positive Raji cells with $\mathrm{P}(\mathrm{BU})_{2}$, sodium butyrate and TGF $\beta$ for the same lengths of time did not significantly affect the percentage of the sub-G1 population that remained at about $10 \%$ for the entire time of treatment. Results similar to those shown in Fig. 1A were obtained when apoptosis was detected by annexin V-FITC binding to phosphatidylserine (data not shown).

In addition, Western blotting analysis of PARP cleavage carried out on the lysates of cells treated as mentioned above, is illustrated in the image of Fig. 1B. It appears that the $89 \mathrm{KDa}$ apoptotic form of the protein was generated in EBV negative Ramos and BL41 cells after incubation with the lytic cycle activators for 24 and 48 hours, respectively. Conversely, only the full form of PARP was 


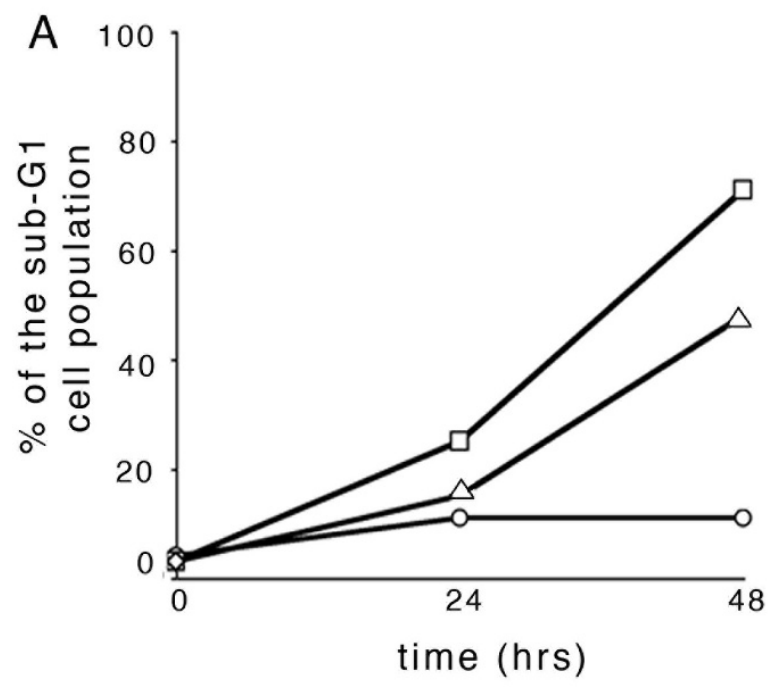

\section{Figure I}

Evaluation of apoptosis in BL cells during exposure to EBV lytic cycle inducing compounds. EBV-positive Raji (circles) and EBV-negative Ramos (squares) and BL4I (triangles) cells were treated with P(BU) ${ }_{2}$, sodium butyrate and TGF- $\beta$ as described in the Methods. At the indicated times (hours), samples were collected and analyzed for (A) cytofluorimetric determinations of sub-GI events after PI staining or (B) immunodetection of native (I I $3 \mathrm{KDa}$ ) and apoptotic (89 KDa) forms of PARP by Western blot. The results are representative of those obtained in at least three independent experiments.

observed in Raji cells even when exposed to $\mathrm{P}(\mathrm{BU})_{2}$ sodium butyrate and TGF $\beta$ for up to 72 hours.

In order to confirm that the different apoptotic response in Raji cells was related to EBV infection, we compared apoptosis in EBV-positive and EBV-negative Akata cells, after treatment with anti-IgG. Table 1 reports the results of a representative experiment as percentages of apoptotic cells (annexin V-positive) and apoptotic plus necrotic (annexin $\mathrm{V}$ and propidiun iodide positive) cells measured by flowcitometry at 0,24 and 48 hours. It is evident that at 24 hours, the fraction of apoptotic cells in the EBVinfected population was about one fifth of that measured in the absence of the virus, indicating that EBV protects

Table I: Evaluation of apoptosis in EBV-positive and EBVnegative Akata cells treated with anti-IgG

\begin{tabular}{lccc}
\hline & $\mathrm{T}^{\mathrm{a}}$ & Ann $\mathrm{V}$ b & Ann $\mathrm{V}+\mathrm{PI} \mathrm{c}$ \\
\hline Akata & 0 & 4 & 5 \\
EBV & 24 & 10 & 12 \\
positive & 48 & 22 & 25 \\
\hline Akata & 0 & 5 & 16 \\
EBV & 24 & 54 & 26 \\
negative & 48 & 8 & 75 \\
\hline
\end{tabular}

a Time of exposure of the cells to anti-lgG

b AnnexinV-positive cells (\%)

c Annexin $V$ plus propidium iodide-positive cells (\%)
Akata cells from IgG cross-linking induced apoptosis. This result was confirmed by the analysis of the values measured at 48 hours indicating that post-apoptotic processes in the EBV-negative cellls resulted in a much larger increment of cells permeable to propidium iodide. Results similar to those shown in the Table were obtained when EBV lytic cycle induction was triggered by $\mathrm{P}(\mathrm{BU})_{2}$, sodium butyrate and TGF $\beta$.

\section{Expression of viral and cellular antiapoptotic genes during EBV lytic cycle induction}

Because EBV-positive Raji cells appeared to be protected from apoptosis despite incubation with multiple EBV lytic cycle inducing compounds, we next examined the levels of expression of both viral as well as cellular antiapoptotic genes. Among the viral genes, LMP1, BHRF1 and BALF1 have been reported to suppress apoptosis. However, BALF1 is not expressed in Raji cells because comprised in the genome deletion that accounts for the replication defect in this strain [25]. Therefore, we analyzed LMP1 and BHRF1 expression in Raji cells collected at different times after addition to the culture of EBV lytic cycle activators. Fig. 2A shows that the immediate early antigen BZLF1 ( $34 \mathrm{KDa}$ ) is rapidly expressed after EBV induction and high levels of the protein are detected in the samples collected after 8 hours of incubation with the inducers. Similarly, the levels of LMP1 protein $(63 \mathrm{KDa})$ dramatically increased after 8 hours of exposure of the cells to $\mathrm{P}(\mathrm{BU})_{2}$, sodium butyrate and TGF $\beta$ and raised further, 
A

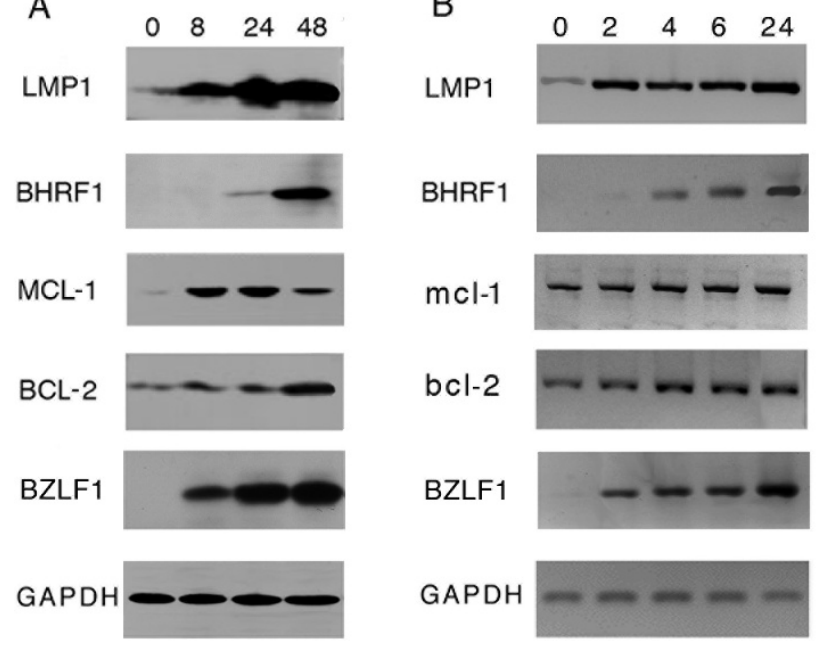

Figure 2

Expression of viral and cellular antiapoptotic genes during EBV lytic cycle activation. (A) Raji cells were exposed to lytic cycle inducing agents as described in the Methods. At the indicated times (hours), (A) cell lysates were resolved by $10 \%$ acrylamide SDS-PAGE, blotted on membrane and probed with specific antibodies; (B) purified RNA was reverse transcribed and cDNA amplified with the specific primers (see Methods).

reaching at 48 hours values about 10 times higher than those measured in latently-infected cells (time 0). Conversely, BHRF1 (17 KDa) was detectable only 24 hours after the addition of the lytic cycle activators and its level increased between 24 and 48 hours.

Because LMP1 induces the cellular anti-apoptotic genes mcl-1 and bcl-2 [13], we examined their expression during EBV lytic cycle activation. The levels of MCL-1 (43 $\mathrm{KDa}$ ) largely increased in the samples collected at 8 and 24 hours but started to decline thereafter, confirming the previously reported transient increment following LMP1 expression. In contrast, BCL-2 levels slightly raised during the first 24 hours of incubation of the cells with the inducers, but in the samples collected at 48 hours, the intensity of the specific band ( $29 \mathrm{KDa}$ ) was about three times higher than that measured in the untreated cells (time 0 ).

To further investigate the time-course and the regulation of the expression of the viral and the cellular anti-apoptotic genes, we measured mRNA levels by RT-PCR to assess whether the up-regulation of LMP1, MCL-1 and BCL-2 proteins was related to increased transcription of the corresponding genes. As it appears from Fig. 2B, a semi-quantitative evaluation of the transcripts indicates that besides EBV lytic genes, also LMP1, Mcl-1 and Bcl-2 expression is regulated at the transcriptional level. However, while LMP1 mRNA levels increased concomitantly with BZLF1 gene expression after 2 hours exposure of the cells to EBV lytic cycle activators, BHRF1 transcripts appeared only after 4 hours. Similar times of incubation of the cells with the inducing agents were necessary to detect a slight but consistent increment of mcl-1 and bcl2 mRNAs.

\section{EBV activation and ROS production}

Variations in the redox state play an important role in modulating cell survival and apoptosis. Therefore, we asked whether the different percentage of apoptotic cells measured in Raji and Ramos exposed to EBV activating compounds, was related to variations in the levels of reactive oxygen species (ROS). To this end, Raji and Ramos cells were incubated with $\mathrm{P}(\mathrm{BU})_{2}$, sodium butyrate and TGF $\beta$ for up to 48 hours. Within this period of time, cell samples were collected, subjected to an oxidation-sensitive probe and analyzed by flowcitometry. Fig. 3A shows that in Raji cells ROS production increased in the first four hours, reached a plateau and augmented to a greater extent between 24 and 48 hours. In contrast, in Ramos cells ROS levels were lower, both at early as well as at late times after the addition to the culture of the inducing agents.

In particular, at 48 hours, the values measured in the EBV negative cell line were about one third of those measured in Raji cells. To further evaluate these results, we analyzed the sub-G1 population in Raji and Ramos cells exposed to the inducers for 48 hours, in the absence or in the presence of $\mathrm{N}$-acetyl cysteine. The bargraph of Fig. 3B indicates that a slightly higher percentage of apoptosis was measured in Raji cells when EBV lytic cycle was induced in the presence of the antioxidant molecule. However, N-acetyl cysteine was not able to reduce apoptosis in Ramos cells, clearly confirming that cell death of EBV-negative cells was not related to higher production of ROS.

\section{Contribution of signal transduction pathways to protection of induced Raji cells from apoptosis}

In order to assess the role played by different signal transduction pathways in protecting induced Raji cells from apoptosis, EBV induction was carried out during exposure of the cells to specific inhibitors of the main signaling molecules known to be activated during the latent and/or the early phases of the lytic EBV infection. Therefore, Raji cells were incubated with $\mathrm{P}(\mathrm{BU})_{2}$, sodium butyrate and TGF $\beta$ for 48 hours in the presence of SB203580, PD98059, SP600125 or Wortmanin, specific inhibitors of p38 MAPK, ERK, JNK and PI3K, respectively or with Bay 117082, inhibitor of NFkB pathway. Thereafter, cells were subjected to cell cycle analysis and the apoptosis measured as the sub-G1 fraction of the cell population. As it 
A

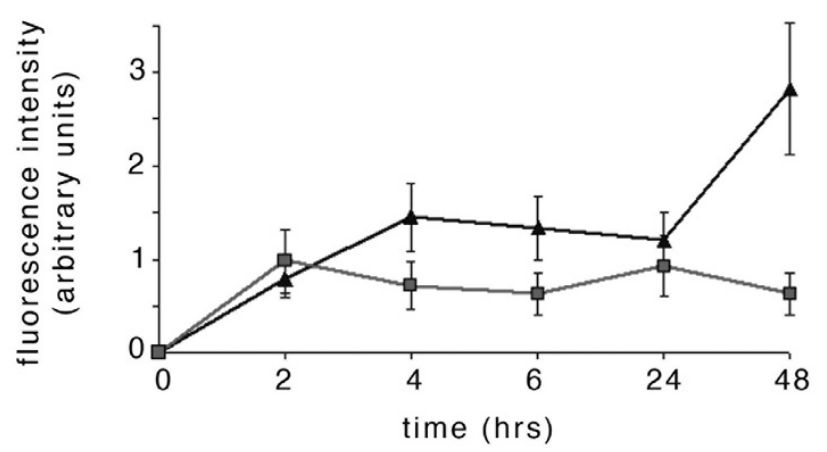

B

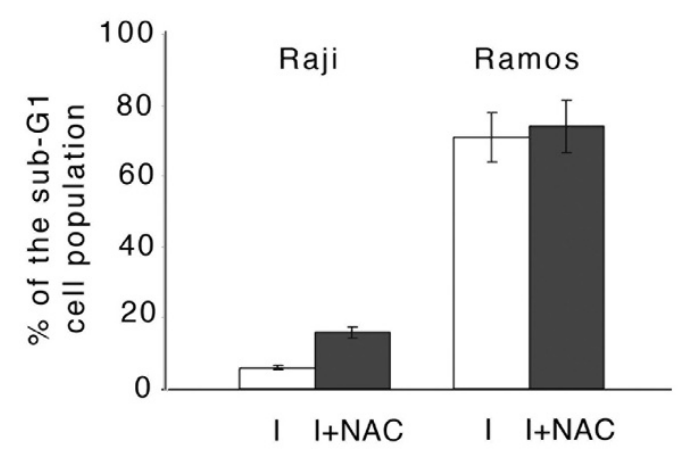

Figure 3

ROS production in Raji and Ramos cells exposed to EBV lytic cycle activators. (A) Cells were treated with $\mathrm{P}(\mathrm{BU})_{2}$, sodium butyrate and TGF $\beta$. At the indicated times, samples of Raji (triangles) and Ramos (squares) were incubated with an oxidation-sensitive probe and the oxidized fluorescent derivative analyzed by flowcytometry; (B) cells exposed to EBV lytic cycle activators in the absence (open bars) or in the presence (filled bars) of $\mathrm{N}$-acetyl cysteine for 48 hours were fixed and PI stained for cytofluorymetric determination of sub-GI events. Error bars are the means \pm $\mathrm{SD}$ of three independent experiments.

appears from Fig. 4A, after exposure for 48 hours to EBV lytic cycle activators about $5 \%$ of Raji cells was characterized by a hypodiploid DNA content. This percentage did not vary significantly when EBV induction was carried out in the presence of agents that inhibit ERK, JNK, PI3K and NFkB pathways. In contrast, the addition of SB203580 at a concentration proved not to be toxic on latently-infected cells, causes a three fold increment of apoptosis, indicating that p38MAPK pathway highly contributed to protect EBV-positive Raji cells from apoptosis. To assess the specificity of p38 signaling cascade in the protective effect on Raji cells, we verified the activation of the different pathways by analyzing the phosphorylation pattern of p38, ERK and JNK, as well as NFkB activity, at different times after exposure of the cells to the inducing agents. As shown in Fig. 4B, the results of Western blots carried out with specific antibodies for p38, ERK, JNK and for the phosphorylated forms of the three MAPKs, indicate that all of them were activated. In particular, phosphorylation of both p38MAPK and ERK strongly increased at 1 hour after induction of EBV lytic cycle; however, the levels of phospho-p38 remained high up to six hours, while phospho-ERK increment appeared to be transient. Moreover, JNK activation occurred at later times (3 hours), but increased thereafter. Experiments carried out to verify the efficacy of p38 inhibitor showed that at the concentration used, SB203580 largely suppressed p38 phosphorilation without affecting JNK or ERK activation (data not shown). In addition, NFkB-DNA binding activity analyzed by EMSA (Fig. 4C) revealed an increment in the signal corresponding to the specific complex indicating that induction of EBV lytic cycle led to prompt and persistent activation of the transcription factor NFkB.

\section{Inhibition of signal transduction pathways and EBV lytic cycle activation}

Because p38 signaling cascade appeared to be involved in the protection of EBV-positive cells from apoptosis when exposed to lytic cycle inducing compounds, we asked whether inhibition of this pathway could affect EBV lytic cycle activation. To assess this point, after a 48 hours incubation of Raji cells with $\mathrm{P}(\mathrm{BU})_{2}$, sodium butyrate and TGF $\beta$ in the presence of either SB203580, PD98059, SP600125, Wortmanin, or Bay 117082, cell samples were fixed on slides and treated for immunofluorescence staining with antibodies recognizing EBV early antigens.

In the panel of Fig. 5A, the results of a representative experiment are shown. The image in a) shows that after 48 hours incubation with lytic cycle inducers, FITC-labeled antibodies detected EA in a large percentage of the cell population as previously reported [26]. EA expression was also detected when lytic cycle induction was carried out in the presence of inhibitors of ERK, JNK, PI3K or NFkB pathway (pictures c, d, e and f).

In contrast, FITC fluorescence signal was absent in the samples of cells incubated with SB203580 (picture b), indicating that activation of $\mathrm{p} 38$ pathway is essential for induction of EBV lytic cycle. To further analyze this result, we measured the expression of BZLF1 and BFRF1, an immediate early and an early antigen, respectively, of EBV lytic program. As it appears from Fig. 5B, after a 48 hours treatment with the inducers in the presence of SB203580, the levels of both BZLF1 and BFRF1 were dramatically reduced as compared to those measured in the absence of the $\mathrm{p} 38$ inhibitor.

\section{Discussion}

In this study we have reexamined the relationship between cell survival and EBV lytic cycle to determine viral factors and/or signal transduction pathways involved in 
A

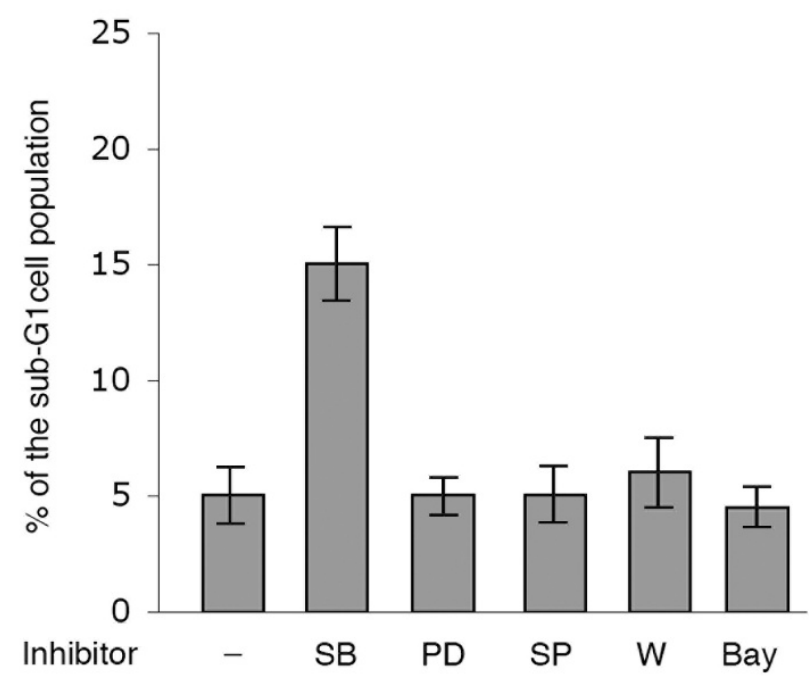

B

C
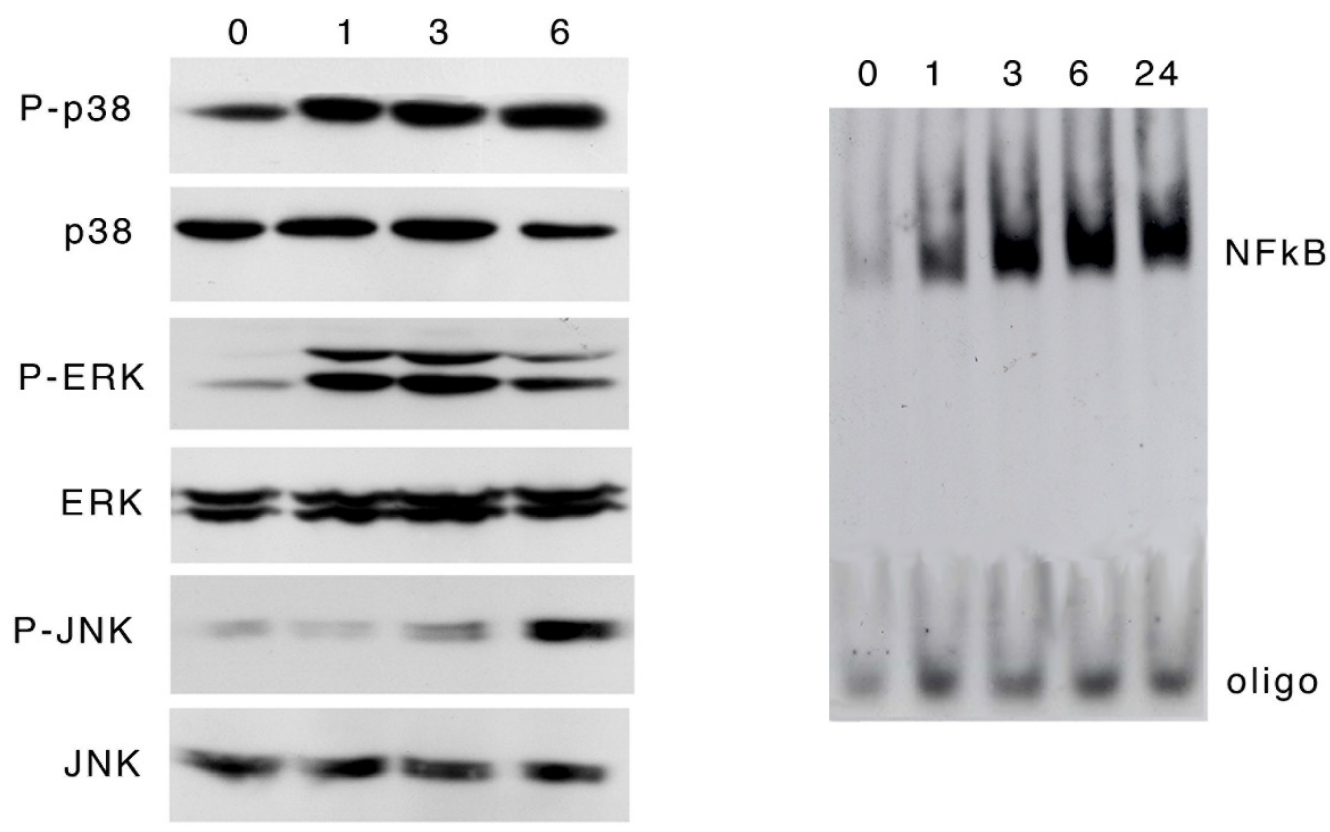

Figure 4

Contribution of signal transduction pathways to cell viability during exposure of Raji cells to EBV lytic cycle activators. (A) Cells were pretreated for 15 min with the specific inhibitors of p38 (SB), ERK (PD), JNK (SP), PI3K (W) or NFkB (Bay) and then exposed to $P(B U)_{2}$, sodium butyrate and TGF $\beta$ as described in the Methods. After 48 hours, cells were fixed and PI stained for cytofluorymetric determination of sub-GI events; $(B)$ cells were incubated with $P(B U)_{2}$, sodium butyrate and TGF $\beta$. At the indicated times (hours), cell lysates were prepared and the phosphorylation pattern of $p 38$, ERK and JNK evaluated by Western blot analysis with the specific antibodies for each protein and its phosphorylated (P-) form; (C) cells were treated as in B. At the indicated times, cell extracts were incubated with a consensus NFkB binding site oligonucleotide (oligo) to evaluate NFkB activity by EMSA (see Methods). 
A

a

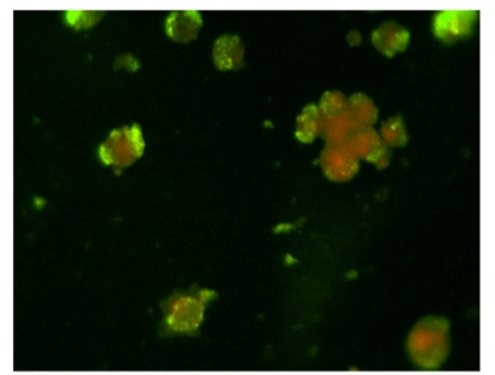

c

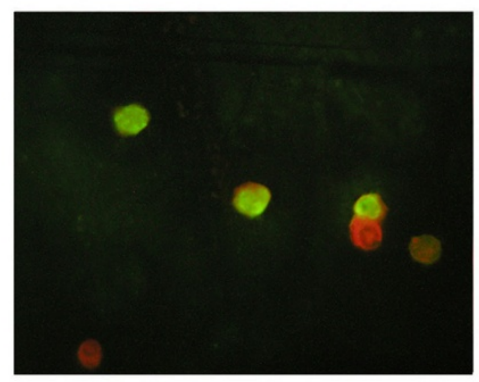

e

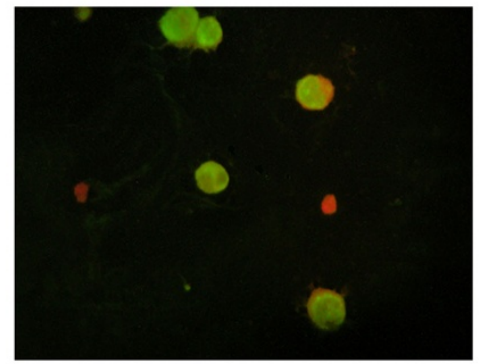

b

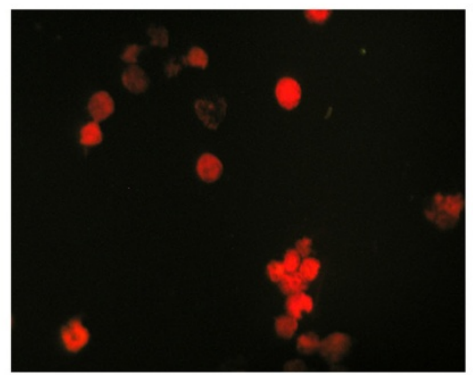

d

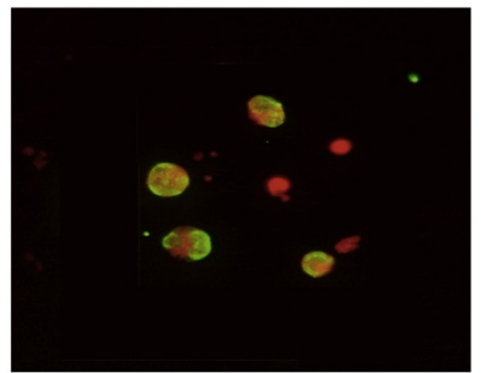

f

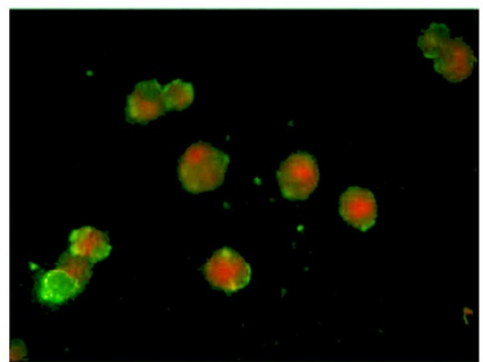

B
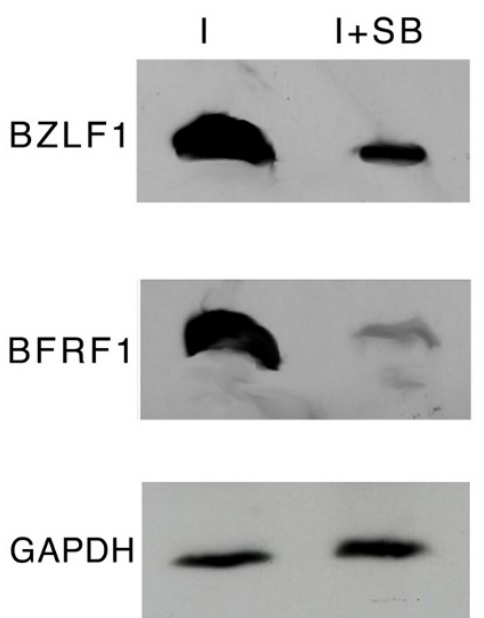

Figure 5

EBV activation in the presence of inhibitors of signal transduction pathways. Raji cells were treated as described in Fig. 4 A. (A) Fixed cells were stained for immunofluorescence analysis with FITC-labelled antibodies specific for EBV early antigens: a) no inhibitor; b), c), d), e), f) plus inhibitor of p38, ERK, JNK, PI3K or NFkB, respectively; (B) Raji cells were exposed to EBV activators in the absence (I) or in the presence (I+SB) of p38 inhibitor. At 48 hours, cell extracts were resolved onto a $10 \%$ acrylamide gel by SDS-PAGE, blotted and probed with BZLFI or BFRFI antibodies. Hybridization with GAPDH antibodies was used as loading control. Specific signal were visualized by ECL.

the protection of EBV-infected cells from apoptosis during viral reactivation. We attempted to investigate this issue in Raji cells that, treated with multiple agents, support a massive induction of an abortive EBV lytic cycle $[25,26]$ characterized by the expression only of the viral IE and $\mathrm{E}$ genes. We show here that in contrast to EBV-negative $\mathrm{BL}$ cell lines, EBV-positive Raji and Akata cells do not die, even after prolonged exposure to the activating agents. These results confirm and strengthen what previously observed; in addition, because late antigens are not expressed in Raji cells, our data exclude the possibility that a late viral product is involved in the protective effect.
ROS have been implicated in the regulation of several cellular functions, including intracellular signaling, transcription activation, proliferation, and apoptosis [27-29]. For what concerns apoptosis, ROS can act as a reactive signal, denature DNA and alter intracellular organelles [3032].

We asked whether treatment of the cells with EBV lytic cycle activators would differently affect ROS levels in EBVpositive and negative BL cells to account for survival or apoptosis. Recently, it has been reported that in vitro EBV infection is associated with ROS production [33]; furthermore, EBV-positive BL have been shown to express high 
levels of MAPK and ROS while EBV-negative BLs exhibit activation of PI3-kinase, but do not have elevated levels of ROS [34]. Our data show that upon exposure to lytic cycle inducing compounds, ROS production remains higher in the EBV-positive as compared with the EBV-negative cells; in addition, pretreatment with $\mathrm{N}$-acetyl cystein (a ROS inhibitor) did not block cell death of EBV-negative BL cells, further indicating that apoptosis occurring in these cells is not related to ROS production.

We next looked for viral factors and signal transduction pathways that could play a role in the protection of EBVpositive cells from apoptosis upon induction of EBV lytic cycle.

Many gamma herpesviruses appear to express their BCL-2 homologues early in the lytic replication cycle, raising the possibility that herpesvirus BCL-2 proteins prolong cell survival to allow production of greater numbers of progeny. Here we show that EBV BCL-2 homologue BHRF1 is expressed only 24 hours after addition of lytic cycle activators, while a dramatic increment of LMP1 is detectable as soon as after 8 hours. Moreover, the transcriptional activation of LMP1 in the lytic cycle results in the up-regulation of the MCL-1 and BCL-2 cellular anti-apoptotic proteins as previously described to occur in LMP1-transfected B lymphocytes [13].

The increment of LMP1 in the early phases of EBV lytic cycle has been previously reported $[35,36]$. However, in contrast to the knowledge of the roles during EBV latency, little is known about the biological significance of LMP1 up-regulation during the lytic cycle of viral replication. It has been reported that loss of LMP1 severely impairs the release of viral particles [37]. Other studies have shown that LMP1 expression inhibits EBV lytic cycle induction and progress [38]. Furthermore, a number of reports indicate that LMP1 expression levels directly affect the ability to stimulate signaling and cell proliferation positively, or to inhibit protein synthesis and induce cytostasis $[39,40]$.

Far from being completely elucidated, it is conceivable that LMP1 up-regulation during EBV lytic cycle induction serves to prepare a suitable cell environment for virus replication by promoting cell survival or by triggering some essential signaling pathways. In this respect, our preliminary results obtained in cells exposed to EBV lytic cycle activators in the presence of LMP1-siRNA indicate that the latter is able to prevent LMP1 up-regulation and increase the percentage of apoptotic cells. Moreover, immunofluorescence experiments carried out on Raji cells exposed to EBV lytic cycle activators confirmed that BZLF1 and high levels of LMP1 are co-expressed in the same cell (data not shown).
We show here that the inhibition of p38 MAPK at the onset of EBV lytic cycle, increases by three fold the percentage of apoptotic Raji cells indicating that p38 phosphorylation largely contributes to protect EBV-positive BL cells during the early phases of EBV activation. It is well known that $\mathrm{p} 38 \mathrm{MAPK}$ as well as NFkB signaling transduction cascades are triggered by LMP1 to inhibit apoptosis and promote cell survival $[10,41]$. Our data indicate an increment of NFkB activity upon induction of EBV lytic phase of infection. However, evidence has been provided showing that treatment of Kaposi's sarcoma-associated herpesvirus (KSHV) or EBV latently-infected lymphocytes with an NFkB inhibitor leads to virus reactivation, suggesting that high NFkB activity levels contribute to maintenance of the viral latency state [42]. It can be hypothesized that the apparent discrepancy between our results and what was previously reported strictly depends on the cellular context. In this respect, treatment of Raji cells with an NFkB inhibitor did not lead to EBV reactivation in Raji cells (unpublished observations).

We also report that inhibition of p38 prevents EBV lytic cycle induction in Raji cells indicating the prominent role that this signaling pathway plays in the process of EBV reactivation. Data have been provided demonstrating that siRNA targeting p38MAPK blocks p38 phosphorylation and BZLF1 expression induced by TPA in EBV-positive epithelial cells [43]. Furthermore p38 inhibition prevented efficient disruption of viral latency by surface IgG crosslinking in Akata cells [44]. Our results, obtained in Raji cells in which the virus is activated with three lytic cycle inducing agents, confirm and extend the essential involvement of p38 activation in the break of viral latency. However, because Raji cells are exposed to multiple agents, each one able to trigger BZLF1 expression, it is likely the effector being downstream of BZLF1 activation.

The immediate early genes BZLF1 and BRLF1 activate one another's transcription and together trans-activate EBV early genes. They both induce phosphorylation of p38 kinase as well as JNK to activate the cellular transcription factor ATF2 [44]. It has also been found that, in contrast to BZLF1, the ability of BRLF1 expression vector to induce lytic EBV infection is markedly reduced by inhibition of either p38 or JNK pathways [44]. From our data it seems conceivable that p38 inhibitor might prevent EBV activation by interfering with $\mathrm{p}$ 38-mediated transcription of BZLF1 by BRLF1. However, the delayed phosphorylation of JNK with respect to the other MAPKs and the evidence that EBV activation occurs also in the presence of JNK inhibitor, indicate that this signal transduction pathway is not involved in the early phases of lytic infection in Raji cells. 


\section{Conclusion}

In conclusion, we report here that at the onset of EBV lytic cycle, p38 MAPK activation plays an important role both for protecting EBV-infected Raji cells from apoptosis, as well as for promoting EBV lytic gene expression. Our data strongly suggest that the increment of LMP1 protein occurring upon EBV reactivation may contribute in a relevant way to both events. Studies are currently carried out to test this hypothesis by elucidating the function of LMP1 up-regulation in the switch from latency to EBV lytic program.

\section{Methods}

Cell culture and treatment with EBV lytic cycle activators EBV-positive Raji and Akata and EBV-negative Ramos, BL41 and Akata, are Burkitt's lymphoma (BL)-derived cell lines. They were cultured in RPMI 1640 medium containing 5\% fetal calf serum (FCS) and antibiotics, in a 5\% CO2 atmosphere and maintained at a cell density of $3.5 \times$ $10^{5} / \mathrm{ml}$.

Cells, at a density of $5 \times 10^{5}$ cells $/ \mathrm{ml}$, were incubated in RPMI 1640/2,5\% FCS with $\mathrm{P}(\mathrm{BU})_{2}$ (Sigma), sodium butyrate (Sigma) and TGF- $\beta 2$ (Genzyme, sp.act. $5 \times 10^{7}$ $\mathrm{U} / \mathrm{mg}$ ) at the final concentrations of $20 \mathrm{ng} / \mathrm{ml}, 2 \mathrm{mM}$ and $0.04 \mathrm{ng} / \mathrm{ml}$, respectively.

In addition, EBV-positive and EBV-negative Akata cells at a density of $2 \times 10^{6} / \mathrm{ml}$ were incubated for 2 hours with $100 \mu \mathrm{g} / \mathrm{ml}$ of anti human IgG (Sigma) and subsequently diluted to $10^{6}$ cells $/ \mathrm{ml}$. At different times, cell samples were collected and analyzed as described below. Cell viability was assessed by trypan blue exclusion.

\section{Cytofluorimetric analysis}

The percentage of non viable cells was determined by FACS analysis after DNA staining with propidium iodide (PI). Cell samples were washed with phosphate buffer saline $(\mathrm{PBS})$ and centrifuged for $5 \mathrm{~min}$ at $300 \times \mathrm{g}$. The cell pellet was fixed for $1 \mathrm{~h}$ at $4^{\circ} \mathrm{C}$ with $70 \%$ ethanol, washed with PBS, and treated for 1 hour with $100 \mu \mathrm{g} / \mathrm{ml}$ of PI and $100 \mu \mathrm{g} / \mathrm{ml}$ RNase. DNA content was assessed by using an Epics Coulter XL flowcytometer. Apoptotic cells (pre-G1 peak) were considered as showing a DNA content less than $2 \mathrm{~N}$. Alternatively, early apoptosis was evaluated by annexin V-FITC apoptosis detection kit (Sigma) that measures annexin $\mathrm{V}$ binding to phosphatidylserine in conjunction with propidium iodide staining, according to the accompaining procedure.

\section{Western blot analysis}

Cells (about 106) were collected and washed with PBS before being lysed in Laemmli buffer. To detect phosphorylated proteins, cells were resuspended in lysis buffer $\left(0,1 \% \mathrm{NaN}_{3}, 1 \mathrm{mM} \mathrm{CaCl}_{2}, 1 \mathrm{mM} \mathrm{MgCl}_{2}, 150 \mathrm{mM} \mathrm{NaCl}\right.$,
$0.5 \mu \mathrm{M}$ aprotinin, $4 \mu \mathrm{M}$ leupeptin, $2 \mathrm{mM}$ PMSF, $10 \mathrm{mM}$ $\mathrm{NaF}, 10 \mathrm{mM}$ iodoacetamide, $1 \mathrm{mM} \mathrm{Na}_{3} \mathrm{VO}_{4}, 1 \%$ Triton-X 100 in PBS) and kept for 30 min on ice. Equal amounts of proteins $(70 \mu \mathrm{g})$, as determined by a modified Lowry assay (RC DC protein assay, BioRad), were resolved on a $10 \%$ acrylamide gel by SDS-PAGE and transferred to PVDF membrane. The blots were incubated with PBS/ $0.1 \%$ Tween $20 / 5 \%$ non fat milk for 1 hour before being probed in the same solution with the following primary antibodies: anti LMP1 (1: 7500, PD Pharmigen), anti BCL-2 (1: 200, Dako), anti PARP (1: 5000, Alexis), anti MCL-1 (1: 200, Santa Cruz), anti BHRF1 (1: 20, kind gift from Dr. Kremmer, GSF-Forschungszentrum, Munich, Germany), anti p38 (1: 500, Santa Cruz), anti P-p38 (1: 500, Cell Signaling), anti ERK and anti P-ERK (both 1: 1000, Cell Signaling), anti JNK (1:1 000, Santa Cruz), anti P-JNK (1: 500, Santa Cruz), anti BZLF1 (1: 100, Argene), or anti BFRF1 (1: 1000, kindly provided by Dr. A. Farina, Dept. of Experimental Medicine, Univ. of Rome "Sapienza", Italy), for 1 hour at $25^{\circ} \mathrm{C}$. The blots were further incubated for one hour with horseradish peroxidase conjugated anti-mouse or anti-rabbit (both 1: 5000, Amersham) or anti rat (1: 10000, Jackson IR). The specific signals were visualized by ECL detection kit (Amersham).

\section{RT-PCR experiments}

Total RNA was isolated from Raji cells as previously described [45]. The specific primers for mcl-1, bcl-2, LMP1 and GAPDH, the PCR conditions and the size of the amplified sequences have been previously reported [45]. PCR products were loaded onto a $1.5 \%$ agarose gels containing $0.5 \mu \mathrm{g} / \mathrm{ml}$ ethidium bromide and visualized under UV light.

\section{Production of Reactive Oxigen Species (ROS)}

Aliquots $\left(2.5 \times 10^{5}\right)$ of untreated cells or cells incubated with EBV lytic cycle inducing compounds in the absence or in the presence of $2.5 \mathrm{mM} \mathrm{N}$-acetyl cysteine, were collected and washed three times in $5 \mathrm{mM}$ HBSS buffer $\mathrm{pH}$ 7.4 before being incubated with $1 \mu \mathrm{M} 5,6$-carbossy-2', $7^{\prime}$ diclorohydro fluoresceine diacetate (DCFH-DA, Molecular Probes, Eugene, OR, USA) for 15' at room temperature. ROS production was analyzed by flowcytometry (EPICS Coulter-XL, FL, USA, excitation: 488 nm, emission: 530 $\mathrm{nm}$ ) by measuring the oxidized fluorescent derivative DCF [46]. The levels of ROS detected in untreated cells or in $\mathrm{H}_{2} \mathrm{O}_{2}$ treated cells have been considered as basal level and positive control, respectively, to determine ROS production following EBV lytic cycle induction.

\section{Inhibition of signal transduction pathways}

Samples of Raji and Ramos cells at a density of $1 \times 10^{6} / \mathrm{ml}$, were incubated for $15^{\prime}$ at $37^{\circ} \mathrm{C}$, in the presence of $10 \mu \mathrm{M}$ SB203580 (SIGMA), $10 \mu \mathrm{M}$ PD98059, $10 \mu \mathrm{M}$ SP600125 or $1 \mu \mathrm{M}$ Wortmanin (all from Calbiochem), specific 
inhibitors of p38 MAPK, ERK, JNK and PI3K, respectively, or with $5 \mu \mathrm{M}$ Bay 117082 (Sigma), inhibitor of NFkB. Cells were then diluted with an equal volume of RPMI 1640 medium containing EBV lytic cycle inducing agents. After 48 hours, cell samples were collected and analyzed by Western blot, flowcytometry and fluorescence microscopy.

\section{Electrophoretic Mobility Shift Assay (EMSA)}

Whole cell extracts were obtained after lysis in a high salt extraction buffer (50 mM Tris- $\mathrm{HCl}$ pH 7.5, $400 \mathrm{mM} \mathrm{NaCl}$, $1 \mathrm{mM}$ EDTA, $1 \mathrm{mM}$ EGTA, 1\% Triton X100, 0.5\% NP40, $10 \%$ glycerol, $2 \mathrm{mM}$ DTT, $2 \mu \mathrm{M}$ aprotinin, $2 \mu \mathrm{M}$ leupeptin, $1 \mathrm{mM} \mathrm{Na}_{3} \mathrm{VO}_{4}, 2 \mu \mathrm{M}$ pepstatin, $1 \mathrm{mM}$ PMSF). $20 \mu \mathrm{g}$ were incubated with $30 \mathrm{fmol}$ of a DIG-labeled (DIG oligonucleotide 3' end-labeling kit, Roche Applied Science) kB DNA probe [47], in a binding buffer (20 mM Tris- $\mathrm{HCl} \mathrm{pH}$ 7.5, 2 mM EDTA, 10\% glycerol) containing $1 \mu \mathrm{g}$ BSA, 0.5 $\mu \mathrm{g}$ poly $\mathrm{d}(\mathrm{I}-\mathrm{C})$, for $20 \mathrm{~min}$ at room temperature. Complexes were resolved by nondenaturing $4 \%$ polyacrylamide gel electrophoresis, transferred to nylon membrane and detected by chemiluminescence (DIG luminescent detection kit, Roche Applied Science).

\section{Fluorescence microscopy}

Treated cells were smeared on slide, fixed and permeabilized with methanol:acetone $(2: 1)$ for $5 \mathrm{~min}$ at $-20^{\circ} \mathrm{C}$ and then stained with FITC-conjugated F6-Ester 2 antibodies recognizing EBV early antigens (EA), as previously described [48]. Slides were mounted with 50\% glycerol in PBS and analyzed with a Leica DM4000 fluorescence microscope equipped with a FX 340 digital camera.

\section{Competing interests}

The authors declare that they have no competing interests.

\section{Authors' contributions}

GM carried out most of the experimental work and contributed to draft the manuscript. GA and ADL equally contributed to the present work by accomplishing cell culture treatments and helping to elaboration of data. LDR carried out cytofluorimetric analysis. EM participated and coordinated the study, compiled and finalized the manuscript. All authors read and approved the final manuscript.

\section{Acknowledgements}

This work has been partially supported by a contribution of the "Istituto Pasteur, Fondazione Cenci Bolognetti", University of Rome "La Sapienza" and by grants from the Italian Ministry of Education, University and Research (MIUR).

We thank Pankaj Trivedi for providing the EBV-positive and EBV-negative Akata cell lines.

\section{References}

I. Rickinson AB, Kieff EB: Epstein Barr Virus Philadelphia, P.A.: Lippincott Williams \& Wilkins; 200I.

2. Li QX, Young LS, Niedobitek G, Dawson CW, Birkenbach M, Wang $F$, Rickinson $A B$ : Epstein-Barr virus infection and replication in a human epithelial cell system. Nature 1992, 356:347-350.

3. Sugden B: An intricate route to immortality. Cell 1989, 57:5-7.

4. Kim KR, Yoshizaki T, Miyamori H, Hasegawa K, Horikawa T, Furukawa M, Harada S, Seiki M, Sato H: Transformation of MadinDarby canine kidney (MDCK) epithelial cells by Epstein-Barr virus latent membrane protein I (LMPI) induces expression of Ets I and invasive growth. Oncogene 2000, I9: I764-I77I.

5. Wang D, Liebowitz D, Wang F, Gregory C, Rickinson A, Larson R, Springer $T$, Kieff E: Epstein-Barr virus latent infection membrane protein alters the human B-lymphocyte phenotype: deletion of the amino terminus abolishes activity. J Virol 1988 , 62:4I73-4I84.

6. Kulwichit W, Edwards RH, Davenport EM, Baskar JF, Godfrey V, Raab-Traub N: Expression of the Epstein-Barr virus latent membrane protein I induces $B$ cell lymphoma in transgenic mice. Proc Natl Acad Sci USA 1998, 95: I 1963-I 1968.

7. Wilson JB, Weinberg W, Johnson R, Yuspa S, Levine AJ: Expression of the BNLF-I oncogene of Epstein-Barr virus in the skin of transgenic mice induces hyperplasia and aberrant expression of keratin 6. Cell 1990, 61:1315-1327.

8. Eliopoulos AG, Young LS: LMPI structure and signal transduction. Semin Cancer Biol 200 I, I I:435-444.

9. Lam N, Sugden B: CD40 and its viral mimic, LMPI: similar means to different ends. Cell Signal 2003, I5:9-16.

10. Soni V, Cahir-McFarland E, Kieff E: LMPI TRAFficking activates growth and survival pathways. Adv Exp Med Biol 2007, 597:173-187.

II. Henderson S, Rowe M, Gregory C, Croom-Carter D, Wang F, Longnecker R, Kieff E, Rickinson A: Induction of bcl-2 expression by Epstein-Barr virus latent membrane protein I protects infected B cells from programmed cell death. Cell I99I, 65: I 107-III5.

12. Rowe M, Peng-Pilon M, Huen DS, Hardy R, Croom-Carter D, Lundgren $E$, Rickinson $A B$ : Upregulation of bcl-2 by the Epstein-Barr virus latent membrane protein LMPI: a B-cell-specific response that is delayed relative to NF-kappa $B$ activation and to induction of cell surface markers. J Virol 1994, 68:5602-56I2.

13. Wang S, Rowe M, Lundgren E: Expression of the Epstein Barr Virus Transforming Protein LMPI Causes a Rapid and Transient Stimulation of the Bcl-2 Homologue Mcl-I Levels in BCell Lines. Cancer Res 1996, 56:4610-4613.

14. Fries KL, Miller WE, Raab-Traub N: Epstein-Barr virus latent membrane protein I blocks p53-mediated apoptosis through the induction of the A20 gene. J Virol 1996, 70:8653-8659.

15. Ai MD, Li LL, Zhao XR, Wu Y, Gong JP, Cao Y: Regulation of Survivin and CDK4 by Epstein-Barr virus encoded latent membrane protein I in nasopharyngeal carcinoma cell lines. Cell Res 2005, I 5( I 0):777-784.

16. Countryman J, Miller G: Activation of expression of latent Epstein-Barr herpesvirus after gene transfer with a small cloned subfragment of heterogeneous viral DNA. Proceedings of the National Academy of Sciences of the United States of America 1985, 82:4085-4089.

17. Takada K, Shimizu N, Sakuma S, Ono Y: trans activation of the latent Epstein-Barr virus (EBV) genome after transfection of the EBV DNA fragment. J Virol 1986, 57:1016-1022.

18. Chevallier-Greco A, Manet E, Chavrier P, Mosnier C, Daillie J, Sergeant A: Both Epstein-Barr virus (EBV)-encoded trans-acting factors, EBI and EB2, are required to activate transcription from an EBV early promoter. Embo J 1986, 5:3243-3249.

19. Kenney S, Kamine J, Holley-Guthrie E, Lin JC, Mar EC, Pagano J: The Epstein-Barr virus (EBV) BZLFI immediate-early gene product differentially affects latent versus productive EBV promoters. J Virol 1989, 63:1729-1736.

20. Rooney CM, Rowe DT, Ragot T, Farrell PJ: The spliced BZLFI gene of Epstein-Barr virus (EBV) transactivates an early EBV promoter and induces the virus productive cycle. J Virol 1989, 63:3109-3116. 
21. Di Renzo L, Avila-Carino J, Klein E: Induction of the lytic viral cycle in Epstein Barr virus carrying Burkitt lymphoma lines is accompanied by increased expression of major histocompatibility complex molecules. Immunol Lett 1993, 38:207-2I4.

22. Luka J, Kallin B, Klein G: Induction of the Epstein-Barr virus (EBV) cycle in latently infected cells by $\mathbf{n}$-butyrate. Virology |979, 94:228-23।.

23. Takada K, Ono Y: Synchronous and sequential activation of latently infected Epstein-Barr virus genomes. J Virol 1989, 63:445-449.

24. Inman GJ, Binne UK, Parker GA, Farrell PJ, Allday MJ: Activators of the Epstein-Barr virus lytic program concomitantly induce apoptosis, but lytic gene expression protects from cell death. $J$ Virol 200I, 75:2400-24I0.

25. Hatfull G, Bankier AT, Barrell BG, Farrell PJ: Sequence analysis of Raji Epstein-Barr virus DNA. Virology 1988, 164:334-340.

26. Mattia E, Ceridono M, Chichiarelli S, D'Erme M: Interactions of Epstein-Barr Virus Origins of Replication with Nuclear Matrix in the Latent and in the Lytic Phases of Viral Infection. Virology 1999, 262:9-17.

27. Bauer G: Signaling and proapoptotic functions of transformed cell-derived reactive oxygen species. Prostaglandins Leukot Essent Fatty Acids 2002, 66:41-56.

28. Gamaley IA, Klyubin IV: Roles of reactive oxygen species: signaling and regulation of cellular functions. Int Rev Cytol 1999, 188:203-255.

29. Sauer $\mathrm{H}$, Wartenberg $M$, Hescheler J: Reactive oxygen species as intracellular messengers during cell growth and differentiation. Cell Physiol Biochem 200I, I I:173-186.

30. Barzilai $A$, Yamamoto $K$ : DNA damage responses to oxidative stress. DNA Repair (Amst) 2004, 3: I I09-1 II5.

31. Bras M, Queenan B, Susin SA: Programmed cell death via mitochondria: different modes of dying. Biochemistry (Mosc) 2005, 70:231-239.

32. Moldovan L, Moldovan NI: Oxygen free radicals and redox biology of organelles. Histochem Cell Biol 2004, I 22:395-4I2.

33. Lassoued S, Ben Ameur R, Ayadi W, Gargouri B, Ben Mansour R, Attia $H$ : Epstein-Barr virus induces an oxidative stress during the early stages of infection in B lymphocytes, epithelial, and lymphoblastoid cell lines. Mol Cell Biochem 2008, 313:179-186.

34. Cerimele F, Battle T, Lynch R, Frank DA, Murad E, Cohen C, Macaron $\mathrm{N}$, Sixbey J, Smith K, Watnick RS, et al.: Reactive oxygen signaling and MAPK activation distinguish Epstein-Barr Virus (EBV)positive versus EBV-negative Burkitt's lymphoma. PNAS 2005, 102: 175-179.

35. Boos H, Berger R, Kuklik-Roos C, Iftner T, Mueller-Lantzsch N Enhancement of Epstein-Barr virus membrane protein (LMP) expression by serum, TPA, or $n$-butyrate in latently infected Raji cells. Virology 1987, 159:161-165.

36. Torii T, Konishi K, Sample J, Takada K: The truncated form of the Epstein-Barr virus LMP-I is dispensable or complimentable by the full-length form in virus infection and replication. Virology 1998, 25 I:273-278.

37. Ahsan N, Kanda T, Nagashima K, Takada K: Epstein-Barr virus transforming protein LMPI plays a critical role in virus production. J Virol 2005, 79:44I5-4424.

38. Prince S, Keating S, Fielding C, Brennan P, Floettmann E, Rowe M Latent membrane protein I inhibits Epstein-Barr virus lytic cycle induction and progress via different mechanisms. J Virol 2003, 77:5000-5007.

39. Kaykas A, Sugden B: The amino-terminus and membrane-spanning domains of LMP-I inhibit cell proliferation. Oncogene 2000, 19:1400-1410.

40. Lam N, Sandberg ML, Sugden B: High physiological levels of LMPI result in phosphorylation of elF2 alpha in Epstein-Barr virus-infected cells. J Virol 2004, 78: I657-I664.

4I. Eliopoulos AG, Gallagher NJ, Blake SM, Dawson CW, Young LS: Activation of the $\mathrm{p} 38$ mitogen-activated protein kinase pathway by Epstein-Barr virus-encoded latent membrane protein I coregulates interleukin-6 and interleukin-8 production. J Biol Chem 1999, 274:16085-16096.

42. Brown HJ, Song MJ, Deng H, Wu TT, Cheng G, Sun R: NF-kappaB inhibits gammaherpesvirus lytic replication. J Virol 2003, 77:8532-8540.

43. Gao $X$, Wang $H$, Sairenji $T$ : Inhibition of Epstein-Barr virus (EBV) reactivation by short interfering RNAs targeting p38 mitogen-activated protein kinase or c-myc in EBV-positive epithelial cells. J Virol 2004, 78: I I798-I I806.

44. Adamson AL, Darr D, Holley-Guthrie E, Johnson RA, Mauser A, Swenson J, Kenney S: Epstein-Barr Virus Immediate-Early Proteins BZLFI and BRLFI Activate the ATF2 Transcription Factor by Increasing the Levels of Phosphorylated p38 and cJun N-Terminal Kinases. J Virol 2000, 74: | 224- 233.

45. Masciarelli S, Mattioli B, Galletti R, Samoggia P, Chichiarelli S, Mearini G, Mattia E: Antisense to Epstein Barr Virus-encoded LMP I does not affect the transcription of viral and cellular proliferation-related genes, but induces phenotypic effects on EBVtransformed B lymphocytes. Oncogene 2002, 21:4166-4170.

46. Royall JA, Ischiropoulos $\mathrm{H}$ : Evaluation of 2',7'-Dichlorofluorescin and Dihydrorhodamine 123 as Fluorescent Probes for Intracellular H2O2 in Cultured Endothelial Cells. Archives of Biochemistry and Biophysics 1993, 302:348-355.

47. Zabel U, Henkel T, Silva MS, Baeuerle PA: Nuclear uptake control of NF-kappa B by MAD-3, an I kappa B protein present in the nucleus. Embo J 1993, I2:201-211.

48. Mattiussi S, Tempera I, Matusali G, Mearini G, Lenti L, Fratarcangeli S, Mosca L, D'Erme M, Mattia E: Inhibition of Poly(ADP. ribose)polymerase impairs Epstein Barr Virus lytic cycle progression. Infect Agent Cancer 2007, 2: 18.
Publish with Bio Med Central and every scientist can read your work free of charge

"BioMed Central will be the most significant development for disseminating the results of biomedical research in our lifetime. "

Sir Paul Nurse, Cancer Research UK

Your research papers will be:

- available free of charge to the entire biomedical community

- peer reviewed and published immediately upon acceptance

- cited in PubMed and archived on PubMed Central

- yours - you keep the copyright
BioMedcentral 